\title{
Les Enregistrements Comme Moyen de Preuve en Droit Congolais et en droit Francais
}

\author{
Jack’s Mbombaka Bokoso ${ }^{1}$
}

\section{Résumé :}

Justice! Ce mot, fort de symboles, incarne l'aspiration de la victime d'une infraction qui souhaite légitimement être entendue dans sa volonté de voir punir le coupable que celle de la personne injustement mise en cause qui attend de la société qu'elle reconnaisse son innocence. Le processus pénal doit alors assurer l'équilibre entre les «deux intérêts également opposés, puissants et sacrés » qui veulent à la fois être protégés. En équilibre, la preuve pénale en forme le « gage». Les enregistrements et vidéos servent aujourd'hui des preuves indiscutables en droit français; le droit congolais les n'en donne pas autant d'importance. Face à cette évidence, devrons-nous continués toujours à mettre de côté cette catégorie des preuves devenues irréversibles et redoutables face à la forte évolution de la technologie au Congo? C'estpourquoi cet article plaide pour une modification rapide du code de procédure pénale congolaise afin que soient intégrés ses armes redoutables.

\begin{abstract}
Justice! This word, full of symbols, embodies the aspiration of the victim of an offense who legitimately wishes to be heard in his will to punish the culprit as that of the unjustly questioned person who expects society to recognize his innocence. The penal process must then ensure a balance between the "two equally opposing, powerful and sacred interests" who want to be protected at the same time. In balance, criminal evidence forms the "pledge". The recordings and videos today serve indisputable evidence in French law; Congolese law does not give them the same importance. Faced with this evidence, will we still have to put aside this category of evidence that has become irreversible and daunting in the face of the strong evolution of technology in the Congo? That is why this article argues for a rapid modification of the Congolese Code of Criminal Procedure so that its dreadful weapons are integrated.
\end{abstract}

1 Jack's MBOMBAKA BOKOSO est assistant à la faculté de droit de l'université de Kinshasa de la République démocratique du Congo;il est aussi apprenant en troisième cycle de la même faculté. Il est Avocat au Barreau près la cour d'appel de Kinshasa/Matete. Il est egalement Secrétaire National chargé de la communication du Parti Réformateur pour un Congo Vivant, parti cher à maitre Aimé KILOLO MUSAMBA. Il est enfin membre de la fondation CONRAD ADENAWERAyant sa représentation en République démocratique du Congo. Mail : mbombakabokoso@gmail.com. 


\section{INTRODUCTION}

En république Démocratique du Congo, la liberté de la preuve en matière pénale demeure un principe général de droit renforcé par une jurisprudence constante ${ }^{2}$. Elle est constituée de l'ensemble de principes directeurs et de règles appropriées ${ }^{3}$ qui déterminent les conditions dans lesquelles la preuve de la commission d'un fait infractionnel par une personne suspectée ${ }^{4}$ doit être recherchée et recueillie, par les organes déterminés par la loi, et administrée devant les juridictions répressives compétentes, dans le respect et limites fixées par

2 C.S.J., R.P. 324, Cass. matière répressive, Kabamba Mupemba c/ le ministère publique et Société Boukin, Arret du 07 octobre 1980, bulletin des Arrêts de la cour suprême de justice (1980-1984), Kin.,p. 83; C.S.J., R.P 324, Cass., matière répressive, kabamba Mupemba c/ le ministère publique, Arrêt, 07 octobre 1980, Bulletin des Arrêts de la cour suprême de justice (2004-2009),T. II, kin. 2010, p. 83.

3 Un principe peut s'entendre d'une norme générale fondatrice ou directrice du droit tandis que la règle est l'application d'un principe. Lire à ce sujet Vergès $M$. E., Les principes directeurs du procès judiciaire, Etude d'une catégorie juridique, Thèse pour le doctorat en droit, Université de Droit, d'Economie et de Sciences d'Aix-Marseille, Faculté de Droit et de Science Politique d'Aix-Marseille, décembre 2000. Cette étude, cependant, à l'instar du constituant congolais, considérant que cette distinction n'est pas si tranchée, distinguera les deux concepts ou en usera indifféremment, le cas échéant. A titre d'exemple, le constituant congolais affirme que le principe selon lequel les personnes poursuivies doivent demeurer libres est la règle, leur détention l'exception; alors qu'il considère l'interdiction de l'emprisonnement pour dettes comme un des principes fondamentaux auxquels il ne peut être dérogé même lorsque l'état de siège ou l'état d'urgence aura été proclamé. Voir Constitution de la République Démocratique du Congo, modifiée par la Loi n ${ }^{\circ} 11 / 002$ du 20 janvier 2011 portant révision de certains articles de la Constitution de la République Démocratique du Congo du 18 février 2006 (Textes coordonnés), Journal Officiel de la République Démocratique du Congo, 52è année, Numéro Spécial, Kinshasa, 5 février 2011, art 17, alinéa premier; art 6,1, litera 6.

4 La présente étude substitue aux termes «présumé auteur de l'infraction » (Voir, notamment, le Code de Procédure Pénale Congolaise, art. 5, 6, 11; l’Ordonnance nº 78-289 du 3 juillet 1978 relative à l'exercice des attributions d'officier et agents de police judiciaire près les juridictions de droit commun, JOZ, 1er août 1978, ${ }^{\circ}{ }^{15}$, arts 97, 103, 105) et « inculpé » (Voir le Code de Procédure Pénale Congolaise, art. 4, 11, 15; Ordonnance $\mathrm{n}^{\circ} 78-289$ du 3 juillet 1978 relative à l'exercice des attributions d'officier et agents de police judiciaire près les juridictions de droit commun, op.cit., art 96) celui de suspect ou de personne suspectée. En effet, ainsi que le relevait le professeur BAYONA BAMEYA, les expressions «présumé auteur de l'infraction » et "inculpé » violent le principe constitutionnel de la présomption d'innocence. Effectivement, « on ne peut concevoir que le délinquant, qui est présumé innocent, soit déjà présumé comme celui qui a commis l'infraction ». De même, le terme " inculpé » " viole le principe constitutionnel de la présomption d'innocence car à ce stade de la procédure, le délinquant est déjà considéré comme étant coupable »si l'on considère, qu'étymologiquement, in culpa se réfère à la faute. Voir Nicolas-AbelBAYONA BA MEYA, La terminologie juridique à l'épreuve de la pratique légale, judiciaire et sociale au zaïre, in Revue de la Faculté de droit, Le droit aux prises avec les réalités socioculturelles, Actes des journées scientifiques organisées par la Faculté de droit, Université Protestante au Congo, lère année, numéro spécial, 1998, p. 13,14. Par conséquent, dans la présente étude, suspect ou personne suspectée est la personne contre laquelle, selon la police ou le parquet, il existe des raisons plausibles de croire qu'elle peut avoir commis ou qu'elle a pu tenter de commettre une infraction et qui est entendue ou commise à comparaitre devant le juge d'instruction, sur réquisitoire introductif du parquet. Voir à ce sujet, par exemple, C. GUERY, Les droits du suspect, in Lemonde.fr, disponible sur http://www.lemon 
la Constitution ${ }^{5}$.D'autant pour dire que ce principe ayant une importance capitale n'est pas réglementé dans notre code de procédure pénal congolais comme c'est le cas dans d'autres pays ${ }^{6}$.

Ainsi, il a été en effet jugé qu'en matière pénale le juge doit baser sa décision sur son intime conviction et peut rechercher la preuve des faits dans tous les éléments de la cause et notamment dans les renseignements inclus dans les procès-verbaux réguliers ${ }^{7}$. Il peut aussi fonder sa conviction de la culpabilité du prévenu sur les résultats de l'examen dactylosco-

de.fr/idees/article/2010/03/12/les-droits-du-suspect-par-christian-guery_1318156_3232.html consulté le 30 octobre 2012. En droit international pénal, au cours d'une audience publique de la CPI, la défense de Monsieur Thomas LUBANGA déplora le fait que ce dernier, avant la confirmation de ses charges, avait été présenté, dans une lettre d'information de la CPI (Newsletter), par le Greffe, comme un accusé. Selon la défense, en effet, LUBANGA, étant présumé innocent, et tant que ses charges n'étaient pas confirmées, ne pouvait être présenté comme un accusé mais, plutôt, comme un suspect. Le juge Jorda approuva la position de la défense de LUBANGA, en affirmant qu'il était tout à fait inadmissible que, dans une lettre d'information de la Cour Lubanga fut présenté comme une personne accusée alors qu'il était présumé innocent et que ses charges n'avaient pas encore été confirmées. Voir International Criminal Court, Situation Democratic Republic of Congo, Case $n^{\circ}$ ICC-01/04-01/6, Transcription $n^{\circ}$ ICC-01/04-01/06-T-30-EN, Open session, Thursday, 9 November, 2006, p.15, lignes 19-25; p. 16, lignes 1-3; p. 19, lignes 7-12. En fait, la discussion susvisée, considérée, au regard du Statut de Rome, confirme le fait que le suspect est, en droit international pénal, la personne présumée innocente et qui doit être traitée comme telle mais contre laquelle il existe des motifs de croire qu'elle a commis un crime relevant de la compétence de la Cour et doit être interrogée (Voir Statut de Rome, op.cit., art 55, alinéa 2.) ou encore la personne présumée innocente mais contre laquelle il existe des motifs raisonnables de croire qu'elle a commis un crime relevant de la compétence de la Cour et qui fait l'objet d'un mandat d'arrêt, qu'elle ait été arrêtée ou non, ou qui fait l'objet d'une citation à comparaître devant la Cour, tant que ses charges n'auront pas été confirmées (Voir Statut de Rome, note, arts58, 61).

5 Le constituant congolais de 2006 utilise la terminologie " accusé ». Voir Constitution de la République Démocratique du Congo, op.cit., art 17, dernier alinéa. Selon le professeur Bayona, l'usage du terme accusé « paraît incorrect dans la mesure où, en droit français, il concerne le prévenu qui est traduit devant la Cour d'Assises juridiction qui ne peut pas être comparée à aucune juridiction analogue au Zaïre ». (Voir BAYONA BA MEYA, Cours de procédure pénale, Kinshasa, Unikin, 1994-1995, p. 17. Toutefois, en droit international pénal, un accusé est le suspect contre lequel le Procureur de la CPI a établi devant la Chambre préliminaire de la Cour l'existence « des preuves suffisantes donnant des motifs substantiels de croire que la personne a commis chacun des crimes qui lui sont imputés » et dont, par conséquent, la chambre confirme les charge et la renvoie " devant une chambre de première instance pour y être jugée sur la base de charges confirmées » (Voir Statut de Rome portant sur la C.P.I, adopté en 2002, art 61, alinéa 7, litera a); art 67). Ainsi la présente étude considère l'accusé ou la personne accusée comme la personne contre laquelle le juge d'instruction a trouvé, à l'issue d'un interrogatoire contradictoire, la personne assistée de son conseil, des indices sérieux et concordants faisant croire que cette personne peut avoir commis ou peut avoir tenté de commettre les faits mis à sa charge et qui, par conséquent, est renvoyée, par une ordonnance motivée de renvoi, devant la juridiction compétente pour y être jugée.

6 Art. 353 al. 2 du code de procédure pénal Français.

7 Léo., 26 octobre 1937, R.J.C.B., 1938, p. 111; Elis., 19 mars 1940, R.J.C.B., 1940, p. 131. 
pique des empreintes relevées sur le lieu $\mathrm{du} \mathrm{vol}^{8}$, à la condition qu'il soit démontré que les empreintes en question sont celles de l'auteur de l'infraction. ${ }^{9}$

S'agissant des enregistrements et vidéos, le législateur congolais n'en fait nullement allusion. La jurisprudence congolaise en fait de même. Et pourtant, ils sont devenus aujourd'hui, face à l'évolution de la technologie, les armes irréversibles et redoutables devant servir le juge pénal au-delà de tout doute raisonnable.

Si au cours d'un procès, le prévenu arrivait a nié systématiquement les faits mis à sa charge, lesquels faits dont il fut enregistré lors de la commission de l'infraction, ne devonsnous pas crédibiliser davantage ses enregistrements aux fins d'établir sa responsabilité pénale?

Ce fut à l'unanimité, la position prise par le juge français dans quelques affaires lui soumît, notamment celle dite de Carrefour, l'affaire infanticide et celle dite du « Testing », trois causes jugées devant la Cour de Cassation française que nous avons tenté d'analyser dans notre point A, avant d'examiner la position prise par le juge congolais dans l'affaire dite de « Kamwina N'sapu » dans le point B.

\section{A. LA POSITION DU JUGE FRANÇAIS SUR LES ENREGITREMENTS VIDEOS}

Ce point analyse dans son première sous point, les deux célèbres affaires rendues par la cour de cassation française dont l'arrêt « carrefour » et l'arrêt « infanticide » et dans son deuxième sous point, l'autre affaire célèbre dite du « Testing ».

\section{Affaires « Carrefour » et "Infanticide »}

Vu l'importance de l'affaire, nous avons choisi de commencer l'analyse de l'arrêt Carrefour bien avant l'analyse de celui dit Infanticide.

\section{Affaire dite de « Carrefour»}

Dans le premier arrêt dit de « carrefour », les faits se présentent comme suit : la société carrefour, ayant constaté une baisse importante du montant de ses encaissements en numéraire dans l'un de ses établissements, avait installé une caméra dans une bouche d'aération pour surprendre, le cas échéant, des employés à la caisse en train de s'emparer des sommes d'argent. Il apparut bientôt que plusieurs employés s'étaient emparé de numéraire et avaient en même temps détruits des tickets de caisse. La société déposa plainte pour abus de confiance et à l'appui remit à la police les films accusateurs. Immédiatement le juge d'instruction inculpa cinq employés qui d'ailleurs passèrent presque tous aux aveux. Mais la question de l'irrégularité des enregistrements se posa. 
La chambre d'accusation de Caen affirma la régularité de la procédure et la chambre criminelle de la cour de cassation française, dans son arrêt rendu le 23 juillet 1992, confirma cette décision au degré d'appel. A l'appui de sa décision, la cour de cassation invoque trois arguments :

Qu'il n'existe aucun texte de procédure qui interdit la production par le plaignant des pièces de nature à constituer des charges contre les personnes visées dans la plainte. Il n'y a pas des raisons de s'inquiéter des preuves qui ont pu être amassées par une victime «à chaud » avant le commencement de la procédure. On ne risque aucun conflit entre parties privées et organes officiels et, en outre, il est important d'amasser aussitôt que possible, voir pendant la commission de l'infraction, les premières preuves ${ }^{10}$.

La chambre criminelle de la cour de cassation française allègue en second lieu que les enregistrements effectués par la victime d'une infraction ne constituent pas des actes de procédure mais des pièces à conviction, donc non susceptibles d'annulation ${ }^{11}$.

Enfin, la chambre criminelle de la cour de cassation française invoque le fait qu'il appartient aux juridictions répressives d'apprécier la valeur des enregistrements au regard des règles relatives à l'administration de la preuve des infractions ${ }^{12}$.

\section{Affaire dite d' «Infanticide »}

Dans la deuxième affaire dite d'" infanticide ", deux époux, parents d'un enfant, vivent séparés de fait. L'enfant disparait. Une information est ouverte du chef d'enlèvement de mineur. La mère de l'enfant disparu raconte au juge d'instruction que son mari lui a avoué, avec force détails, qu'il a assassiné l'enfant. Une inculpation d'assassinat est prononcée cotre le père, inculpation fondée sur ces déclarations corroborés par des enregistrements de conversation entre les deux parents versé au dossier par l'épouse.

Devant la chambre d'accusation, le père invoque la nullité des enregistrements. La chambre d'accusation rend cependant un arrêt de renvoi en cour d'assises que le père frappe aussitôt de pourvoi. Il soutient dans son pourvoi qu'il y a eu stratagème et atteinte à la vie privée et donc obtention d'une preuve à l'aide d'une infraction; et par voie de conséquence, il ya violation du principe du procès équitable. La chambre criminelle rejette le pourvoi du père en invoquant divers arguments : «Que les cassettes d'enregistrement sont des pièces à conviction n'ayant que la valeur d'indices de preuve et ne constituent pas des actes de l'information susceptibles d'être annulés $»^{13}$.

En outre, la chambre criminelle de la cour de cassation a décidé, à diverses reprises, qu'il n'existe aucune disposition légale qui permet aux juges répressifs d'écarter les moyens de preuve produits par les parties au seul motif que ces moyens auraient été obte-

10 José-Marie TASOKI MANZELE, Procédure pénale Congolaise, Paris, 2011. p. 283.

11 José-Marie TASOKI MANZELE, note ${ }^{\circ} 10$.

12 José-Marie TASOKI MANZELE, note ${ }^{\circ} 10$.

13 Marie TASOKI MANZELE, note n 10, p. 284. 
nus de manière illégale. ${ }^{14}$ Pour la jurisprudence française, l'essentiel est donc que la preuve puisse être discutée contradictoirement, en fait à l'audience, dans le cadre d'un procès équitable.

\section{L'arrêt du « Testing»}

L'arrêt du 11 juin 2002 est extrêmement original. En effet, l'association SOS Racisme soupçonnait des tenanciers de discothèques de n'accueillir que des clients non maghrébins. Elle organisa une opération dite de « testing », d'origine anglo-saxonne. Divers personnes furent réparties en petit groupes composés les uns d'individus d'origine magrébine et les autres d'individus d'origine européenne, et elles se présentèrent à l'entrée de divers discothèques. Lestenanciers refusèrent l'entrée des personnes d'origine magrébine.

Le parquet lança des poursuites pour discrimination dans la fourniture d'un service à raison de l'origine raciale ou ethnique à l'encontre des tenanciers. Les juges du fonds prononcèrent des relaxes pour illicéité du « testing ", défaut de loyauté et attente aux droits de la défense. Par son arrêt du 11 juin 2002, la chambre criminelle de la cour de cassation française casse l'arrêt entrepris rendu le 05 juin 2001. Elle invoque classiquement l'indifférence de l'origine illicite ou déloyale de la preuve et rappel, « qu'il appartient au juge du fond d'en apprécier la valeur probante après débat contradictoire ». Voyons aprésent, la position du juge congolais en la matière.

\section{B. LA POSITION DU JUGE CONGOLAIS SUR LES ENREGISTREMENTS VIDEOS}

Les juges congolais se sont largement confrontés à la difficulté de cerner la place des enregistrements vidéos recueillis à l'insu des prévenus, par la police judiciaire congolaise des agents commis aux services de renseignement pendant l'instruction de l'affaire dite de « Kamwina N'sapu » version Kinshasa, jugée devant le Tribunal Militaire de Garnison de Kinshasa/Gombe.

\section{Faits de la cause dans l'affaire « Kamwina N'sapu » version Kinshasa}

Ayant été accusé d'avoir commis plusieurs attaques terroristes dans la ville de Kinsha$\mathrm{sa}^{15}$ dont celle dans la nuit du 08 au 09 Mars 2017, à la onzième rue résidentielle sur le poste

14 Crim. 15 juin 1993, Bull. Crim. n²10, Dalloz 1994, p. 163, note C. MASCALA, 6 avril 1994, bull.crim n¹36, GP, 1994, II, p. 489, note J.P DOUCET; Crim., 30 mars 1999, Dalloz, 2000, p. 391, note T. GARE.

15 Les prévenus ci-après,BAKENGA TUJIBIKILE MUTANGILAY Ferdinand, MPUTU NKONGOLO Alphonse alias Ali, NGANDU PANUKUPA TSHIMANGA Gustave alias PAPA ZAMBO, MUKENGESHAY KAPUKU Daniel, TSHIMANGA TSHIMANGA Ben alias Bene, MUKADI MUTOMBO Christian, NGALAMULUME KAPUMBU Erick, BAKAJIKA MULENDA JeanJacques alias John Jackson, TUDIAKUILA BAKATUNIENGELA Roger alias Bingo, MAZAN- 
de police du point chaud de Limete, en face de la permanence de l'Union pour le Developpement et le Progrès Sociale (UDPS) en sigle, parti politique du feu Etienne TSHISEKEDI WA MULUMBA; celle commise dans la nuit du 16 au 17 juin 2017 à la prison centrale de Makala, celle commise en date du 09 au 10 juin au sous-commissariat de la PNC/MontAmba et au parquet de grande instance de Kinshasa/Matete; celle avortée du 24 juin 2017 à la prison Militaire de Ndolo et enfin celle commise le 29 juin 2017 au commissariat Urbain de la police nationale/FUNA et de la maison communale de KALAMU; après enquête et leur arrestation par les agents de l'ordre, les prévenus ont tous été interrogés par la police sont presque tous passés aux aveux.

A la suite de cet interrogatoire, une caméra cachée fut placée à leur insu dans la salle pour filmer, le cas échéant enregistrer les dépositions des prévenus mis à charge aux fins de le surprendre à l'audience publique du tribunal de céans tenu à l'enceinte de la prison Militaire de Ndolo au cas où ils arrivaient tout simplement à nier les procès-verbaux de leur propre interrogatoire.

Le ministère publique, après avoir transmis les pièces y afférentes, remis en suite le film accusateur d'interrogatoire dans lequel tous les prévenus cités dans cette cause passèrent aux aveux et reconnurent avoir participer aux attaques ayant créé trouble et désolation dans la ville de Kinshasa. Brandi à l'audience, la question de l'irrégularité des enregistrements se posa.

\section{Discutions en droit sur les enregistrements vidéos dans l'affaire "Kamwina N'sapu »}

Ici, il y a lieu d'analyser de prime à bord, la position des parties prévenus et celles des parties civiles (1), et enfin, la décision rendue par le Tribunal de céans (2).

GA MAZANGA François Mazarin alias bin Kamia, NTUMBA MBUYI Romain, alias baba Mona Kebe, KABAMBA KALEWU Francky, LUMENGA LUGENI Reagan, KASHAMA KABATUSUILA Eduard alias Eddo, KABASELE KALUILA Jean, NTUMBA NTUMBA Clément Aaron, NTUMBA NTUMBA Papy, KUPA KWA NZAMBI LUKANYI Dieudonné alias Dieudo, MPOY MUKENDI Placide, KABUPWE KAMWANGE Isaac, KAPINGA BWANYA FATUMA alias Fatu, KALALA LUKETA Kalvin, KASONGA MUKALA Gabriel alias Djibril, BIAYA MUKANZA NTITA Augustin alias Ntala NASHAKALE KATAPA Nancy, NAMBU KATAPA Anny, TUJIBIKILA NGANDU constant Alias Costa, BAKAMANA KENAKUDIKA George, MUB EN GAY BETUKUMESU Prospère alias Chambuyi, MOUKENGE MOUKENGE Paul alias cordon Paul, KABANGU KAZADI Chambuyi Pascal, BEYA TSHILENGI Gecko, TSHIANYIMA BATUBENGA Joseph alias Israël Isalela, KANYONGA DIKUBAKUBA Don de Dieu, TSHIMBOMBO LUKULA Simon, KABASU NKUFULU Séraphin, Crim. 15 juin 1993, Bull. Crim. n²10, Dalloz 1994, p. 163, note C. MASCALA, 6 avril 1994, bull.crim n¹36, GP, 1994, II, p. 489, note J.P DOUCET; Crim., 30 mars 1999, Dalloz, 2000, p. 391, note T. GARE. 
1. Position des parties prévenus et des parties civiles

a) Position des prévenus

Pour la défense, outre le fait de tous nier, ils soutiennent que les enregistrements vidéos tels que recueillis par le ministère public ne l'ont pas été fait en directe, aux dates, jours et heures, dans différents sites dont les attaques ont été perpétrées, capable de prouver le déroulement des celles-ci site après site. Que selon elle, ses enregistrements vidéos ont été par contre recueillis quelques mois après les attaques, en plaine interrogatoire des prévenus lesquels seraient fortement torturés pendant la période de leur garde à vue et n'ayant pas d'autres choix que d'avouer les faits. Ils prirent en effet au juge de céans de ne porter aucun égard aux preuves obtenus par des moyens déloyals et illégals.

b) Position des parties civiles et de l'organe puirsuivante

Les conseils des parties civiles et l'organe poursuivant ${ }^{16}$ soutinrent la légalité de cette procédure et ont à l'appui évoqués les trois jurisprudences étrangères ci-dessus, trachées par la cour de cassation française pour assoir leurs argumentaires. Mais l'argument principal dont s'est servi le juge de la cour de cassation française dans le premier arrêt dit de « carrefour » selon lequel il n'existe aucun texte de procédure qui interdit la production par le plaignant des pièces de nature à constituer des charges contre les personnes accusées, a été l'un des arguments principaux évoqués par les conseils des parties civiles dans son ensemble aussi longtemps qu'en droit congolais d'aujourd'hui comme ce fit en droit français d'hier, il n'existe aucune loi interdisant dans son arsenal juridique la production des enregistrements vidéos récoltés à l'insu du prévenu; et que par conséquent, ils prièrent le tribunal de céans de faire droit à leurs demande et de le déclarer recevable et fonder.

Ils prièrent à la même occasion le tribunal de céans de retenir compte aussi comme moyen de preuve, les aveux judiciaires contenus dans les procès-verbaux de tous les prévenus tant dans l'instruction juridictionnelle que pré juridictionnelle; notamment celui fait par le prévenu KABANGU KAZADI CHAMBUYI Pascal à l'audience publique du 27 février 2018 affirmant que le prévenu MAZANGA MAZANGA Mazarin alias Bin Kamia était venu chez lui en date du 16 mai 2017 accompagné de beaucoup des combattants dont notamment la prévenue KAPINGA BWANYA FATU Alias FATUMA en guise de préparatif des attaques de la prison centrale.

16 Maitre Jack's MBOMBAKA BOKOSO pour le compte de 12 parties civiles ci-après :le Commissaire principal BANIMBA MBUMBA, KINIENGA LUNDU,FALO USENI Isaac, Blandine MFUSI MUMPINI veuve MUGENI KAHENGA, KAPIMBA KABIMA, BANZETUKA KWEBANA, NDUAYA MUNALA, MBUKU MASTAKI, MOKE MATUNGA, TSHIMENA MUTOMBO, LIBANZA BOTEMBE et MAYELE AYEL NKUMU, le Bâtonnier National MATADIWAMBA pour le compte de la République démocratique du Congo, Maitre PENGO TUZOLANA pour le compte de la partie civile KASONGO MULWA et Maitre SENGA MUTEBA pour le compte de la partie civile MUSAMBILE WA MUSAMBILE. 
Outre les aveux, ils prièrent aussi le tribunal de céans de tenir compte des témoignages de MUKENGE KABANGU PEPE confirmant avoir héberger chez lui les assaillants MPUTU NKONGOLO et BAKENGA TUJIBIKILE, tous venus du Kasaï où ils étaient, pour installer le mouvement insurrectionnel KAMWINA NSAPU à Kinshasa. Les parties civiles prirent également au tribunal de tenir compte des témoignages de la Femme du major KASEKE, l'un des assaillants décédé sur le théâtre des opérationsà la prison centrale ${ }^{17}$, à charge du prévenu MPOY MUKENDI Placide qui selon elle, était venu prendre son mari le 16 mai 2017 pour ce rendre au lieu préparatoire de l'attaque de la prison centrale de MAKALA.

Ils prirent enfin au tribunal de céans, de donner crédit aux relevés téléphoniques qui ont attestés la communication permanente des prévenus, confirmant les aveux judiciaires contenus dans les procès-verbaux qui garde la totalité de leurs valeurs probantes que la loi leur attache.

\section{Position du Tribunal}

Dans son jugement rendu le 13 décembre 2018, le Tribunal Militaire de Garnison de Kinshasa/Gombe rejette les arguments de la défense et dit les enregistrements vidéos lui soumis recevables et fondés; selon le Tribunal, faisant sienne la position du juge Français, déclara qu'il n'existe aucune loi de procédure en droit congolais qui interdit la production par le ministère public des vidéos de nature à constituer des charges contre les personnes accusées. En effet dans sa motivation, il estime qu'à défaut par les 36 prévenus poursuivis dans la présente cause de ne donner aucune disposition légale qui interdirait une telle procédure après leurs brillantes plaidoiries et après lecture minutieuse de la législation congolaise en la matière par le Tribunal, cela laisse à croire que cette procédure reste conforme et corresponde tant au principe de la liberté que celui de la loyauté en matière de recherche des preuves de l'infraction.

Cette décision est d'autant la première en droit congolais à avoir tranchée positive sur la légalité des enregistrements vidéo, preuve devenue aujourd'hui, face à l'évolution rapide de la technologie, irréversible et redoutable.

Les parties prévenues ont néanmoins manifesté à l'occasion, leurs volonté sur le banc, d'aller en appel contre cette décision qu'elles estiment injuste, laquelle selon elles, les juges ne pouvaient tirés des vidéos obtenus à l'insu de prévenus par des voie illégales, la conviction qu'il existe des indices sérieux de culpabilité qui du reste, les a amena sans raison, à condamner les prévenus à des peines allant de 20 ans jusqu'à la peine de mort.

17 Trouvé mort au matin du 16 au 17 juin 2017 à l'enceinte de la prison centrale de MAKALA après échange des tirs avec les forces loyalistes, les noms de plusieurs assaillants jugés par le tribunal de garnison de Kinshasa/Gombe ont été retrouvés dans la liste trouvée dans la poche du Major KASEKE qui jadis fut dans la force loyaliste mais par ses convictions personnelles se décida de rejoindre le camp des assaillants. 


\section{CONCLUSION}

Nous voici arriver à la fin de notre étude qui a consistée à l'examen de la loyauté de la preuve pénale face aux enregistrements vidéo en droit congolais, preuves devenues aujourd'hui redoutable sous d'autres cieux, notamment en droit français.

Pour y parvenir, nous avons à l'occasion analyser trois jugements rendus par les juges Français en la matière notamment dans l'affaire dite de Carrefour, Infanticide et Testing et un seul jugement rendu par le juge Congolais dans l'affaire Kamwina N'sapu.

En effet, si à l'absence de la loi, la jurisprudence française a tranchée favorable, c'est-àdire confirmer la loyauté des enregistrements vidéo et introduit par après celui-ci dans son code de procédure pénal, cela n'est pas le cas en droit congolais aujourd'hui. En effet, à l'absence d'une disposition légale crédible, il n'existait bien avant l'affaire Kamwina N'sapu en 2018, aucune décision judiciaire crédible faisant foi, décision déjà confirmée par la cour de cassation Congolaise si non dans son ancienne version par la cour suprême de justice ou encore celle rendue par un Tribunal Militaire quelconque, devant éclairé la religion du juge Congolais lorsqu'il s'agit de trancher sur la procédure ayant abouti à la récolte de la preuve par les enregistrement vidéos.

Cela c'est sans surprise. Car, non seulement à l'époque de l'élaboration du code de procédure pénale congolaise, par manque de la modernisation, un tel moyen de preuve ne pouvait être concevable en République démocratique du Congo, mais aussi et surtout que ses instruments (vidéo et camera) n'existaient absolument pas dans le pays. Et si par impossible l'on pourrait le voir, ce fut dans une classe bien réservée et non à la portée de tous comme c'est le cas aujourd'hui.

Bref, nous jugeons positive l'évolution du droit Congolais et saluons en même temps la position prise par le tribunal Militaire de garnison de Kinshasa/Gombe dans l'affaire lui soumise, car elle a le mérite d'éclairer la procédure pénale congolaise sur cette question précise liée à l'étude de la preuve recueillie par enregistrement vidéo et nous pensons en même temps qu'il y a l'urgence de faire exactement comme le fit le législateur français qui, en d'épit des décisions judicaire, a clairement inséré celui-ci dans son code de procédure pénale.

Voilà pourquoi, cette étude plaide pour la modification rapide du code de procédure pénale congolaise à fin que les enregistrements et vidéos, soient insérés dans le code de procédure pénal Congolaise et revêtir de la valeur probante beaucoup plus supérieur que certains procès-verbaux que la loi accorde une force probante beaucoup plus particulière ${ }^{18}$, de sorte que une fois produit devant un juge avec les images de l'accusé, que cela facilite davantage le travail du tribunal.

18 L'article 75 du code de procédure pénale congolaise accorde une valeur suprême à certains procèsverbaux établis par certains agents de la police judiciaire jusqu'à la preuve littérale contraire, soit encore jusqu'à leurs inscription en faux. C'est le cas des procès-verbaux établis par les agents de la douane, soit par ceux de l'Office Congolaise de Contrôle. 


\section{BIBLIOGRAPHIE}

\section{TEXTES JURIDIQUES}

Convention des Nations-Unies du 10 décembre 1984 contre la torture et autres peines ou traitements cruels, inhumains et dégradants, adoptée et ouverte à la signature, à la ratification et à l'adhésion par l'Assemblée générale dans sa résolution 39/46 du 10 décembre 1984, entrée en vigueur le 26 juin 1987, ratifié par l'Ordonnance-loi n 89-014 du 17 février 1989, Journal officiel n5 du 1er mars 1989, p. 8, voir aussi journal officiel, numéro spécial, 5 décembre 2002;

Déclaration universelle des droits de l'homme, adoptée et proclamée par l'Assemblée générale des Nations-Unies, Résolution 217 A (III), Paris, 10 décembre 1948, Bulletin officiel du Congo Belge 1949, p 1206;

Pacte international relatif aux droits civils et politiques, adopté et ouvert à la signature, à la ratification et à l'adhésion par l'Assemblée générale des NationsUnies dans sa résolution 2200 A (XXI) du 16 décembre 1966, entré en vigueur le 23 mars 1976, ratifié par.la RDC le 1er novembre 1976, voir Journal officiel, numéro spécial, 5 décembre 2002;

Code de procédure pénale Française, version en vigueur au 11 avril 2011, disponible sur http://www.le gifrance.gouv.fr/affichCode.do?cidTexte=LEGITEXT0000060711 54 consulté le 11 octobre 2012;

Constitution de la République Démocratique du Congo, modifiée par la Loi n $11 / 002$ du 20 janvier 2011 portant révision de certains articles de la Constitution de la République Démocratique du Congo du 18 février 2006 (Textes coordonnés), Journal Officiel de la République Démocratique du Congo, numéro Spécial, Kinshasa, 5 février 2011;

Décret du 30 janvier 1940 portant Code pénal tel que modifié jusqu'au 31 décembre 2009 et ses dispositions complémentaires, Ministère de la Justice, RDC, 2010;

Décret du 06 août 1959 portant Code de procédure pénale congolais, Journal officiel de la République Démocratique du Congo en ligne, disponible sur http://www.leganet.cd/Legislation/Droit\%20Judic iaire/D.6.8.1959.ccp.htm consulté le 11 octobre 2012;

Loi n 06/018 du 20 juillet 2006 modifiant et complétant le Décret du 30 janvier 1940 portant Code pénal congolais, Journal officiel, numéro spécial, 25 mai 2009;

Loi nº 06/019 du 20 juillet 2006 modifiant et complétant le Décret du 6 août 1959 portant Code de procédure pénale congolais, Journal officiel, numéro spécial, 25 mai 2009;

Loi n ${ }^{\circ} 11 / 006$ du 25 juin 2011 relative à la ratification de l'Ordonnance-loi n ${ }^{\circ}$ 10/002 du 20 août 2010 portant Code des douanes, disponible sur http://www.leganet.cd/Legislation/Dfiscal/Douanes/Loi. 11.006.25.6.2011.htm consulté le 12 octobre 2012;

Loi n ${ }^{\circ} 11 / 008$ du 09 juillet 2011 portant criminalisation de la torture, disponible sur http://www.leganet .cd/Legislation/DroitPenal/Loi.11.008.9.7.2011.htmconsul té le 11 octobre 2012;

Décret-loi constitutionnel du 29 septembre 1960 relatif au pouvoir judiciaire, M.C. $n^{\circ} 4$ du 31/1/ 1961, p. 29;

Ordonnance $\mathrm{n}^{\circ}$ 78-289 du 3 juillet 1978 relative à l'exercice des attributions d'officier et agents de police judiciaire près les juridictions de droit commun, JOZ, 1er août 1978. 


\section{JURISPRUDENCE}

Affaire KAMWINA N'SAPU enrôlée sous RP 986/17/RMP : 6687/17/KIP devant le tribunal militaire de Garnison de Kinshasa/Gombe.

Affaire carrefour, arrêt du 23 octobre 1992 rendu par la Cour de cassation française.

Affaire infanticide, Arrêt de 04 mars 1993 rendue devant la Cour de cassation française;

Affaire Testing, l'Arret du 11 juin 2002 rendu devant la cour de cassation française;

C.S.J., R.P. 324, Cass. Matière répressive, Kabamba Mupemba c/ le ministère publique et Société Boukin, Arret du 07 octobre 1980, bulletin des Arrêts de la cour suprême de justice (1980-1984), Kinshasa; février, 2010.

C.S.J., R.P 324, Cass., matière répressive, kabamba Mupemba c/ le ministère publique, Arrêt, 07 octobre 1980, Bulletin des Arrêts de la cour suprême de justice (2004-2009)

Léo., 26 octobre 1937, R.J.C.B., 1938, p. 111; Elis., 19 mars 1940, R.J.C.B., 1940, p. 131.

Ière institut., Ruanda-Urundi, app., 11 octobre 1951, R.J.C.B., 1952, p. 115

R.J.C.B., 1930, p. 157.

Crim.15juin 1993, Bull. Crim. n²10, Dalloz 1994, p. 163, note c. mascala, 6 avril 1994, bull.crim n '136, GP, 1994, II, p. 489, note J.P DOUCET; Crim., 30 mars 1999, Dalloz, 2000, p. 391, note T. GARE.

Crim. 15 juin 1993, Bull. Crim. n²10, Dalloz 1994, p. 163, note c. mascala, 6 avril 1994, bull.crim n '136, GP, 1994, II, p. 489, note J.P DOUCET; Crim., 30 mars 1999, Dalloz, 2000, p. 391, note T. GARE.

43. C.S.J., R.P. 311, 15/4/1980, inédit;

44. C.S.J., R.P. 396, 7/10/1980, inédit;

45. C.S.J., R.P. 40; 7/2/1973, Bull. 1974, p. 38;

46. C.S.J., R.P. 47, 4/4/1973, Bull. 1974, p. 90;

47. C.S.J., R.P. 47, 4/4/1973, Bull. 1974, p. 90;

48. C.S.J., R.P. 628 du 11/12/1985, in B.A. 2002, p. 106;

49. C.S.J., R.P. 64 et 68, 15/5/1974, Bull? 1975, p. 159, R.J.Z. 1975, p. 107;

50. C.S.J., R.P. 107, 3/4/1974, Bull. 1975, p. 62, R.J.Z. 1977, p. 66;

51. C.S.J., R.P. 64 et 68, 15/5/1974, Bull. 1975, p. 159, R.J.Z. 1975, p. 107;

52. C.S.J., R.P. 94, 20/2/1975, Bull. 1976, p. 38;

53. C.S.J., R.P. 97, 28/1/1976, Bull. 1977, p. 20;

54. C.S.J., R.P. 97, 28/1/1976, Bull. 1977, p. 20;

55. C.S.J., R.P. 97, 28/1/1976, Bull. 1977, p. 20;

56. C.S.J., R.P.A 16 du 12/05/1972; in B.A. 1972, p. 64;

57. C.S.J., R.P.A 16 du 12/05/1972; in B.A. 1972, p. 64;

58. C.S.J., R.P.A. 13, 7/7/1972, Bull. 1973, p. 125, R.J.Z. 1978, p. 77;

59. C.S.J., R.P.A. 46, 19/5/1977, Bull. 1978, p. 40;

60. C.S.J., R.P.A. 46, 19/5/1977, Bull. 1978, p. 40;

61. C.S.J., R/P. 171, 18/3/1975, Bull. 1976, p. 98. 


\section{DOCTRINE}

AKELE ADAWA Pierre, Le citoyen justicier, La justice privée dans l'Etat de droit, Kinshasa, 2002,. BOUZAT P.,La loyauté dans la recherche des preuves, 1964.

FOFE DJOFE MMALEWA J.P., La question de la preuve devant le Tribunal Pénal International pour le Rwanda, le cas Cyangungu, Paris, 2006; P. 201.

GUIDICELLI-DELAGE G. et MATSOPOULOU H., Les transformations de l'administration de la preuve pénale : perspectives comparées Allemagne, Belgique, Espagne, Etats-Unis, France, Italie, Portugal, Royaume-Uni, Paris, 2003; p. 61.

TASOKI MANZELEJosé-Marie, Procédure pénale Congolaise, Paris, 2011. p. 283.

BAYONA BA MEYA, La terminologie juridique à l'épreuve de la pratique légale, judiciaire et sociale au zaïre, in Revue de la Faculté de droit, Le droit aux prises avec les réalités socioculturelles, Actes des journées scientifiques organisées par la Faculté de droit, Université Protestante au Congo, 1ère année, numéro spécial, 1998, p. 13,14.

JACQUES G. \& SOHIE A., Jurisprudence des Bahemba, des chefferies Yambula, Niembo, Mambze et Muhona du territoire de Kongolo, in Bulletin des tribunaux coutumiers, Société d'Etudes Juridiques du Katanga, Elisabethville, juillet-août-septembre 1963;

Vergès M. E., Les principes directeurs du procès judiciaire, Etude d'une catégorie juridique, Thèse pour le doctorat en droit, Université de Droit, d'Economie et de Sciences d'Aix-Marseille, Faculté de Droit et de Science Politique d'Aix-Marseille, décembre 2000.

$B O L Z E P$. « Le droit à la preuve contraire en procédure pénale », Thèse en vue de l'obtention du grade de docteur en droit, Université de Nancy 2, Faculté de droit, sciences économiques et gestion, décembre 2010.

CAPDEPON Y., Essai d'une théorie générale des droits de la défense, Thèse pour le doctorat en droit, Ecole doctorale, Université Montesquieu-Bordeaux IV, Septembre 2011. 